\title{
Qualidade de Moirões de Eucalipto Tratados Comercializados em Três Municípios do Espírito Santo
}

\author{
Juarez Benigno Paes ${ }^{1}$, Lucas Recla Lombardi², José Tarcisio da Silva Oliveira ${ }^{1}$, \\ Luciana Ferreira da Silva ${ }^{1}$, Lorenzo Lube dos Santos ${ }^{3}$
}

${ }^{1}$ Departamento de Ciências Florestais e da Madeira - DCFM, Universidade Federal do Espírito Santo - UFES, Jerônimo Monteiro/ES, Brasil

${ }^{2}$ Departamento de Engenharia Florestal - DEF, Programa de Pós-graduação em Ciência Florestal, Universidade Federal de Viçosa - UFV, Viçosa/MG, Brasil

${ }^{3}$ Departamento de Engenharia Civil - DEC, Programa de Pós-graduação em Engenharia Civil, Universidade Federal do Espírito Santo - UFES, Vitória/ES, Brasil

\begin{abstract}
RESUMO
A pesquisa consistiu na análise da qualidade de moirões de eucalipto tratados, comercializados nos municípios de Alegre, Jerônimo Monteiro e Cachoeiro de Itapemirim, no Estado do Espírito Santo. Para sua realização, compararam-se a distribuição, a penetração e a retenção do preservante arseniato de cobre cromatado (CCA) nos moirões, com os parâmetros norteadores das Normas Brasileiras Regulamentadoras. Por meio de reações colorimétricas, foram avaliadas a distribuição e a penetração do cobre nas peças; por espectrofotometria de absorção atômica, quantificou-se a retenção dos elementos do preservativo empregado. De posse desses parâmetros, verificou-se que os moirões comercializados nos três municípios atendem à normatização quanto à distribuição do produto preservativo. No entanto, observouse que alguns estabelecimentos não satisfazem as exigências quanto às penetrações e retenções mínimas exigidas pela normatização brasileira. Observaram-se as melhores retenções nos moirões comercializados nos municípios de Alegre e Jerônimo Monteiro.
\end{abstract}

Palavras-chave: madeira tratada, análise da qualidade, Eucalyptus sp., CCA.

\section{Quality of Treated Eucalypts Fence Posts Marketed in Three Municipalities of the State of Espirito Santo, Brazil}

\begin{abstract}
The purpose of this research was to examine the quality of treated eucalypts fence posts marketed in the municipalities of Alegre, Jerônimo Monteiro and Cachoeiro de Itapemirim, State of Espirito Santo, Brazil. To this end, the distribution, penetration and retention of chromate copper arsenate (CCA) water borne preservative in fence posts were compared to the parameters of the Brazilian Regulatory Standard Norms. Distribution and penetration were assessed by means of colorimetric reactions, while retention of the preservative product was assessed by atomic absorption spectrophotometry. With these parameters, it was found that the fence posts marketed in the three municipalities meet the standards for the preservative product distribution. However, it was possible to notice that some commercial establishments do not meet the requirements for minimum penetration and retention required by the Brazilian standards. The best retentions were found in fence posts marketed in the municipalities of Alegre and Jerônimo Monteiro.
\end{abstract}

Keywords: treated wood, quality analysis, Eucalyptus sp., CCA. 


\section{INTRODUÇÃO}

Desde os primórdios da civilização, a madeira tem sido um material fundamental para a existência do ser humano, seja na habitação, nas construções em geral, no mobiliário, nas embalagens e em outros setores. A madeira, embora seja considerada renovável, é um recurso finito, e requer, para que sua perpetuidade seja garantida, uma utilização racional, embasada no princípio do rendimento sustentável, ou seja, o uso racional da madeira com garantia de suprimento para as gerações futuras.

Em função dessa sustentabilidade, surge a necessidade da busca por novas alternativas para suprir a demanda de madeira. No Brasil, o gênero Eucalyptus surgiu como uma opção para atender às necessidades do mercado, por causa da escassez de madeiras nobres na Região Centro-Sul e da legislação brasileira vigente. $\mathrm{O}$ uso do eucalipto para a confecção de peças de madeira tratada se deve a sua disponibilidade no mercado, ao rápido crescimento florestal e às características tecnológicas conhecidas, estudadas e melhoradas.

A dificuldade de obtenção de espécies nativas de alta resistência à degradação biológica obrigou o homem a utilizar outras menos duráveis, principalmente aquelas de rápido crescimento, provenientes de reflorestamentos. Na medida em que a maioria dos reflorestamentos foi realizada com espécies florestais dos gêneros Eucalyptus e Pinus, a utilização da madeira dessas árvores para moirões e outros usos tornou-se prática comum para os produtores rurais. Mas, em virtude da baixa resistência da madeira dessas espécies a organismos xilófagos, há a necessidade de tratálas, para aumentar sua vida útil em serviço, bem como reduzir a demanda por madeira de espécies nativas e o impacto sobre as florestas nativas (Farias Sobrinho et al., 2005; Paes et al., 2005).

Para o tratamento químico da madeira, existem os processos industriais e os não industriais, que podem empregar produtos oleossolúveis ou hidrossolúveis. Nesse contexto, Freitas (2002) cita que, no Brasil, o arseniato de cobre cromatado (CCA) e o borato de cobre cromatado (CCB) são os produtos hidrossolúveis mais empregados no tratamento industrial da madeira roliça de eucalipto destinada a moirões.

No Brasil, a produção anual de moirões de eucalipto tratados é da ordem de 40 milhões de unidades, representando $65 \%$ da madeira tratada (Geraldo, 2010). Contudo, a expansão da utilização da madeira tratada, de modo geral, conduziu ao surgimento de novas usinas de preservação da madeira, fato esse que dificulta o controle dos órgãos de fiscalização, podendo, assim, acarretar a produção e o comércio de madeiras tratadas de forma inadequada. Diante do exposto, pode-se indagar se as madeiras tratadas disponíveis no mercado realmente estão atendendo às especificações técnicas das normas brasileiras.

O controle de qualidade da madeira tratada é feito pela determinação da distribuição, da penetração e da retenção de preservativos na madeira. A penetração e a retenção do preservativo são influenciadas por características relacionadas à madeira e ao processo de tratamento utilizado (Freitas, 2002).

Paes (1991) citou que a retenção e a penetração são os parâmetros básicos para a avaliação de eficiência do tratamento preservativo. A penetração é determinada pela profundidade da camada tóxica que protege a madeira. A retenção é um parâmetro quantitativo e refere-se à quantidade de produto preservativo retido num determinado volume de madeira.

A qualidade das madeiras tratadas está diretamente ligada à retenção, à penetração e à distribuição dos preservativos, fatores esses que dependem basicamente da espécie da madeira, do preservativo e do processo de tratamento utilizado. A Norma Brasileira Regulamentadora - NBR 9480 da Associação Brasileira de Normas Técnicas - ABNT (2009) estabelece que, em função da exposição à qual os moirões de eucalipto tratados com preservativos hidrossolúveis estão sujeitos, a retenção mínima seja de $6,5 \mathrm{~kg}$ de ingredientes ativos por metro cúbico de madeira e a penetração ocorra em todo o alburno e na porção penetrável do cerne.

No Estado do Espírito Santo, bem como em outras unidades da Federação, é preocupante a qualidade da madeira preservada, uma vez que as empresas existentes são de pequeno porte, não são cadastradas na Associação Brasileira de 
Preservadores de Madeira (ABPM) e não possuem acompanhamento técnico; dessa forma, o controle da qualidade da madeira fica sob a responsabilidade das próprias empresas.

Em função das premissas levantadas, objetivouse analisar a qualidade dos moirões de eucalipto tratados, comercializados nos municípios de Alegre, Jerônimo Monteiro e Cachoeiro de Itapemirim, no Estado do Espírito Santo.

\section{MATERIAL E MÉTODOS}

\subsection{Amostragem dos moirões adquiridos}

Os moirões analisados foram obtidos em estabelecimentos comerciais nos municípios de Alegre (estabelecimentos 1 e 2), Jerônimo Monteiro (estabelecimento 3) e Cachoeiro de Itapemirim (estabelecimentos 4, 5 e 6). Empregaram-se três moirões de cada estabelecimento comercial, com diâmetro entre 8 e $12 \mathrm{~cm}$, e comprimento de 2,20 m, os quais foram tomados aleatoriamente das pilhas. Os estabelecimentos foram selecionados em função do local de tratamento da madeira, sendo utilizados moirões oriundos de usinas de tratamento distintas.

Após a obtenção dos moirões, foram retirados dois discos de $2,5 \mathrm{~cm}$ de espessura a $50 \mathrm{~cm}$ da base da peças (posição 1, região de afloramento em peças instaladas no solo), no meio do comprimento (posição 2) e a $10 \mathrm{~cm}$ do topo da peça (posição 3) (Figura 1), nas dependências do Laboratório de Biodeterioração da Madeira (LBM) do Departamento de Ciências Florestais e da Madeira (DCFM) do Centro de Ciências Agrárias (CCA) da Universidade Federal do Espírito Santo (UFES).

Um dos discos destinou-se ao ensaio de distribuição e penetração, e o outro ao ensaio de retenção, com a finalidade de verificar a qualidade do tratamento recebido ao longo do comprimento das peças (Figura 1). Cada disco foi identificado conforme a peça e o estabelecimento comercial.

\subsection{Distribuição e penetração do produto preservativo utilizado}

Uma vez que o arseniato de cobre cromatado (CCA) e o borato de cobre cromatado (CCB) são os preservativos hidrossolúveis mais empregados no tratamento de moirões (Freitas, 2002), a identificação do produto preservativo utilizado para imunizar os moirões foi executada com base na presença ou não de boro nas peças tratadas. O procedimento seguiu a NBR 6232 da ABNT (1973), sendo aplicada sobre a superfície de um disco, retirado de cada moirão, uma solução de álcool polivinílico e iodeto de potássio, que revela, quando em contato com o boro, uma coloração azulada.

Descartada a presença do boro (CCB), a penetração e a distribuição do CCA tiveram como base o cobre presente na madeira tratada. Para tanto, foi feita a análise colorimétrica, seguindose as recomendações da norma citada. Para essa determinação, aplicou-se nos discos uma solução de cromo-azurol S, que revela uma coloração azul escuro intensa na presença do cobre.

Para as determinações da penetração, foram demarcados aleatoriamente sobre os discos dois diâmetros perpendiculares entre si, a partir dos quais foram medidas, com uma régua milimétrica, as penetrações. $\mathrm{O}$ valor médio das medições foi utilizado para avaliar a penetração do elemento cobre em cada posição nos moirões. A distribuição foi avaliada com base nas médias das medidas de penetração ao longo do comprimento de cada moirão.

\subsection{Determinação da retenção do CCA}

Para a determinação da retenção do preservativo, foi efetuada a digestão das amostras provenientes dos discos obtidos a $50 \mathrm{~cm}$ da base dos moirões (Figura 2), seguindo-se a metodologia descrita por Wischer (1976 apud Moreschi, 1985). A metodologia consta da determinação da massa e do volume das amostras, e da incineração em mufla para obtenção das cinzas e dos sais metálicos, a $500-550{ }^{\circ} \mathrm{C}$, tendo sido, posteriormente, adicionados $3 \mathrm{~mL}$ da mistura dos ácidos sulfúrico, perclórico e nítrico, todos concentrados, nas proporções de 7:2:1, às cinzas obtidas pela incineração.

A digestão acelerada das amostras ocorreu pelo aquecimento da mistura dos ácidos e cinzas, em chapa aquecida, até que a mistura ficasse límpida, sendo diluídas as soluções ácidas obtidas em água destilada a volumes fixos de $100 \mathrm{~mL}$. 
Após a diluição, os frascos foram identificados e enviados para a realização das leituras por espectrofotometria de absorção atômica, para quantificar os componentes do produto preservativo nas amostras. A quantificação do elemento cobre foi realizada no Laboratório de Análises de Fertilizantes, Águas, Minérios, Resíduos, Solos e Plantas (LAFARSOL) da UFES. No Laboratório de Espectrometria Atômica da Universidade Federal de Viçosa, realizaram-se as quantificações de cromo e arsênio.

A partirdos dados obtidos pela espectrofotometria e do volume das amostras de madeira, os cálculos de retenção foram efetuados de acordo com a Equação 1, apresentada por Paes (1991).

$R=\frac{F \times L \times F d \times 10^{-3}}{V}$

em que: $\mathrm{R}=$ retenção do elemento na madeira ( $\mathrm{kg}$ i.a. $\left.\mathrm{m}^{-3}\right) ; \mathrm{F}=$ fator estequiométrico empregado para transformação dos elementos químicos para óxidos (cobre $\times 1,2518=\mathrm{CuO}$; cromo $\times 1,9230=\mathrm{CrO}_{3}$; arsênio $\left.\times 3,0680=\mathrm{As}_{2} \mathrm{O}_{5}\right) ; \mathrm{L}=$ leitura obtida do espectrofotômetro $(\mathrm{mg} / \mathrm{L})$; Fd = fator de diluição necessário para as leituras no espectrofotômetro; $\mathrm{V}=$ volume das amostras utilizadas nas análises $\left(\mathrm{cm}^{3}\right)$.

\subsection{Análise dos resultados}

Para a análise dos resultados, empregou-se o delineamento inteiramente casualizado com arranjo fatorial, em que foi avaliada a qualidade de moirões tratados comercializados em nove estabelecimentos comerciais localizados no sul do Estado do Espírito Santo.

A penetração do CCA foi avaliada em três posições nas peças (base, meio e topo) e a retenção, em três posições no disco retirado na região de afloramento dos moirões (externa, mediana e interna).

Nas análises e na avaliação dos ensaios realizados, empregou-se o teste de Duncan, a 5\% de probabilidade, para os fatores e a interação detectados como significativos pelo teste de F.

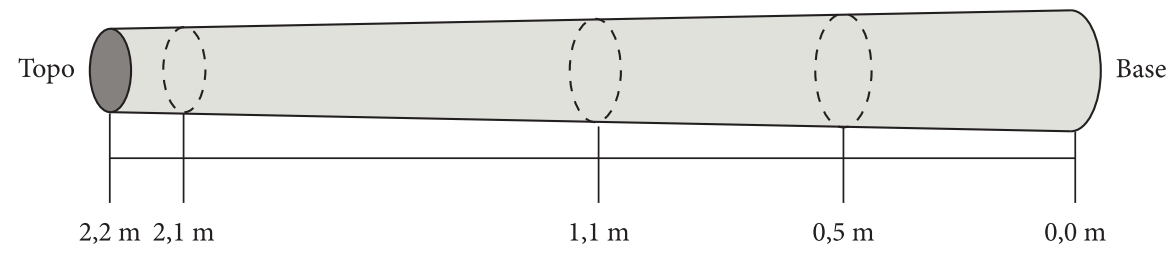

Figura 1. Posições no moirão em que foram retirados os discos para análises químicas.

Figure 1. Positions in the fence post where the discs were taken for chemical analysis.

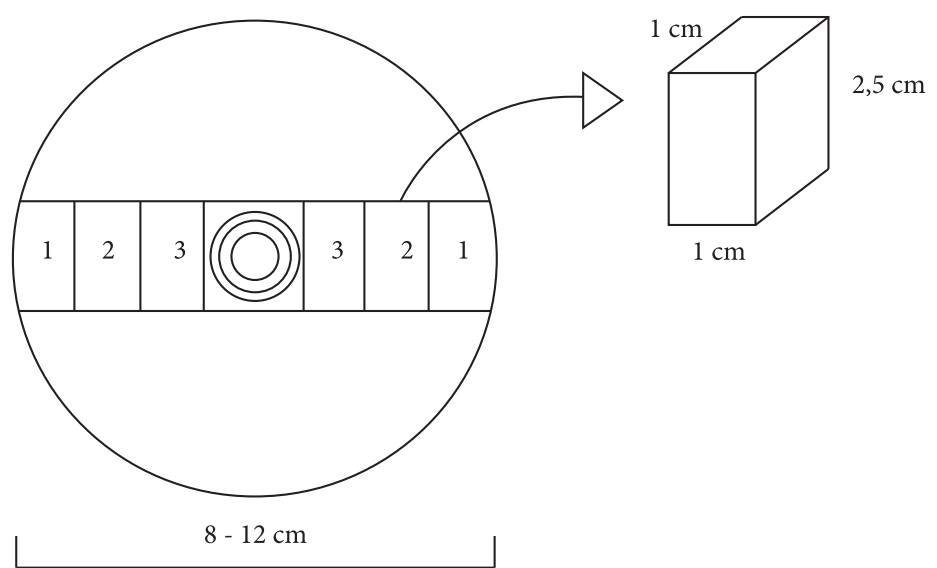

Figura 2. Posições no disco e dimensões das amostras retiradas para a análise química. Figure 2. Position on disk and dimensions of samples obtained to chemistry analysis. 


\section{RESULTADOS E DISCUSSÃO}

\subsection{Distribuição e penetração do CCA nas peças analisadas}

A análise dos discos obtidos dos moirões mostrou que todos os tratamentos foram realizados com o emprego do CCA, uma vez que não houve reação colorimétrica para o boro. Notou-se que o preservativo penetrou em todas as peças, mas de forma diferente em cada uma delas (Tabela 1). A diferença entre o processo de tratamento de cada empresa é ressaltada nos diferentes níveis de penetração. Contudo, a espécie de eucalipto utilizada também pode ter influenciado nos valores obtidos.

Torres et al. (2011) observaram que a distribuição em peças de eucalipto tratadas pelo método da substituição da seiva é influenciada pela inversão das peças durante o processo. Tal ocorrência não foi verificada, indicando que as peças foram tratadas em autoclave, em processo industrial. Neste, a menor penetração é obtida no meio das peças; porém, a penetração do CCA nos moirões foi homogênea, havendo pequenas diferenças entre as posições analisadas de cada moirão. Uma exceção foi observada para as peças provenientes dos estabelecimentos 3 e 5 , em que foram verificadas diferenças de penetração de $8,33 \mathrm{~mm}$ (meio e topo das peças) e de $5,17 \mathrm{~mm}$ (meio e base das peças), respectivamente.

As maiores penetrações foram observadas em amostras obtidas nos moirões adquiridos no estabelecimento 3 (Jerônimo Monteiro) e as menores, no material procedente do estabelecimento 6 (Cachoeiro de Itapemirim).
Ressalta-se que apesar de a NBR 9480 (ABNT, 2009) estipular que a penetração do produto preservativo deve ser total no alburno (madeiras de folhosa), a penetração do preservante na madeira, segundo as considerações de Galvão (1968) e Paes (1991), pode ser aceita como satisfatória quando superior a $10 \mathrm{~mm}$.

Os valores que deram origem às penetrações médias (Tabela 1) foram analisados estatisticamente, tendo o teste $\mathrm{F}$ indicado efeito significativo para a variável penetração, apenas entre os estabelecimentos. Os resultados do teste de médias aplicado e suas comparações encontram-se na Tabela 2.

A análise da penetração de CCA nos moirões comercializados no estabelecimento 3 revelou valor superior às demais (Tabela 2). Também a penetração do preservante observada na madeira proveniente do estabelecimento 1 foi superior aos valores obtidos para as peças procedentes do estabelecimento 6 (Cachoeiro de Itapemirim). As penetrações obtidas nos moirões adquiridos nos estabelecimentos $5,4 \mathrm{e}$ 2 não diferiram estatisticamente, sendo esses valores intermediários aos observados nos moirões obtidos nos estabelecimentos 1 e 6 (Tabela 2).

\subsection{Retenção do produto preservativo nas peças analisadas}

Os valores de retenção para cada estabelecimento e posição no disco foram analisados estatisticamente, tendo o teste de $\mathrm{F}$ indicado efeito significativo em nível de $1 \%$ de probabilidade entre a posição em que a amostra foi retirada no disco e entre estabelecimentos comerciais pesquisados; em nível de 5\%, houve efeito significativo para a interação entre posição de retirada da amostra e estabelecimento comercial. As

Tabela 1. Penetração média $(\mathrm{mm})$ do preservativo CCA em três posições nos moirões de eucalipto oriundos de cada estabelecimento.

Table 1. Average penetration ( $\mathrm{mm}$ ) of CCA preservative in three positions on eucalypts fence posts for each establishment.

\begin{tabular}{cccc} 
Estabelecimento & \multicolumn{3}{c}{ Penetração no moirão $(\mathbf{m m})$} \\
\cline { 2 - 4 } & Base & Meio & Topo \\
\hline 1 & $20,91 \pm 8,22$ & $21,50 \pm 7,53$ & $22,25 \pm 7,30$ \\
3 & $18,41 \pm 3,26$ & $17,50 \pm 3,54$ & $18,33 \pm 3,32$ \\
4 & $27,17 \pm 7,47$ & $23,83 \pm 5,49$ & $32,16 \pm 12,39$ \\
5 & $18,16 \pm 2,90$ & $18,08 \pm 3,64$ & $19,16 \pm 4,02$ \\
6 & $22,75 \pm 10,64$ & $17,58 \pm 3,62$ & $19,25 \pm 5,43$ \\
\hline
\end{tabular}


médias das retenções foram analisadas pelo Teste de Duncan a $5 \%$ de probabilidade e constam nas Tabelas 3, 4 e 5 .

Observou-se, ao analisar o efeito da posição na retenção, que essas posições diferiram entre si, apresentando a posição 1 (externa) os maiores valores de retenção e a posição 3 (interna), os

Tabela 2. Comparações entre médias de penetração (mm) do preservativo CCA nos moirões para cada estabelecimento comercial.

Table 2. Comparisons among average of penetration $(\mathrm{mm})$ of CCA preservative on fence posts for each commercial establishment.

\begin{tabular}{|cc|}
\hline Estabelecimento & Penetração(mm) \\
\hline 3 & $27,72 \pm 8,43^{\mathrm{a}}$ \\
\hline 1 & $21,55 \pm 7,68^{\mathrm{b}}$ \\
\hline 5 & $19,86 \pm 6,55^{\mathrm{bc}}$ \\
\hline 4 & $18,47 \pm 3,49^{\mathrm{bc}}$ \\
\hline 2 & $18,08 \pm 3,36^{\mathrm{bc}}$ \\
\hline 6 & $14,52 \pm 3,68^{\mathrm{c}}$ \\
\hline
\end{tabular}

Médias seguidas pela mesma letra não diferem estatisticamente pelo teste de Duncan $(\mathrm{p} \geq 0,05)$.

Tabela 3. Comparações entre retenções de CCA $\left(\mathrm{kg}\right.$ i.a.m $\mathrm{m}^{-3}$ ) nas posições no disco.

Table 3. Comparisons among CCA retentions $\left(\mathrm{kg}\right.$ a.i. $\left.\mathrm{m}^{-3}\right)$ at positions in disck.

\begin{tabular}{|c|c|}
\hline Posição no disco & $\begin{array}{c}\text { Retenção CCA } \\
\left(\mathrm{kg} \text { i.a. } \mathbf{m}^{-3}\right)\end{array}$ \\
\hline 1 - Externa & $10,63 \pm 3,81^{\mathrm{a}}$ \\
\hline 2 - Mediana & $8,12 \pm 5,49^{b}$ \\
\hline 3 - Interna & $3,46 \pm 4,49^{c}$ \\
\hline
\end{tabular}

Médias seguidas pela mesma letra não diferem estatisticamente pelo teste de Duncan $(\mathrm{p} \geq 0,05)$.

Tabela 4. Comparações entre médias de retenção do CCA ( $\mathrm{kg}$ i.a. $\mathrm{m}^{-3}$ ) nos moirões provenientes dos diferentes estabelecimentos.

Table 4. Comparisons among averages to CCA retention (kg i.a. $\mathrm{m}^{-3}$ ) of fence posts from different establishments.

\begin{tabular}{|c|c|}
\hline Estabelecimento & $\begin{array}{c}\text { Retenção CCA } \\
\left(\mathrm{kg} \text { i.a.m }{ }^{-3}\right)\end{array}$ \\
\hline 3 & $11,52 \pm 0,98^{\mathrm{a}}$ \\
\hline 4 & $9,34 \pm 4,01^{\mathrm{ab}}$ \\
\hline 1 & $8,12 \pm 0,98^{\mathrm{bc}}$ \\
\hline 5 & $7,75 \pm 0,51^{\mathrm{bc}}$ \\
\hline 2 & $5,35 \pm 1,57^{\mathrm{cd}}$ \\
\hline 6 & $2,39 \pm 0,98^{\mathrm{d}}$ \\
\hline
\end{tabular}

Médias seguidas pela mesma letra não diferem estatisticamente pelo teste de Duncan $(\mathrm{p} \geq 0,05)$. menores valores; note-se que estes se apresentaram menores do que o mínimo recomendado pela norma NBR 9480 (ABNT, 2009). Isto indica que, caso a madeira tratada desenvolva rachaduras superiores a $2 \mathrm{~cm}$ de profundidade, poderá expor a organismos xilófagos material não protegido devidamente pelo tratamento, ocasionando substituições prematuras das peças tratadas.

$\mathrm{Na}$ Tabela 4, verifica-se o efeito do local de aquisição das peças (estabelecimento ou indústria de tratamento) na retenção do CCA. Nota-se que os moirões adquiridos no estabelecimento 3 apresentaram as maiores médias de retenção, seguidos pelos provenientes do estabelecimento 4 (Cachoeiro de Itapemirim). As retenções nos moirões adquiridos nos estabelecimentos 1 e 5 foram semelhantes e não diferiram daqueles existentes nos estabelecimentos 4 e 2 . A retenção média atestada nos moirões do estabelecimento 2 foi semelhante àquela dos moirões provenientes do estabelecimento 6.

A análise do efeito da retenção em cada posição no disco nos moirões adquiridos nos diferentes estabelecimentos (Tabela 5) indicou que, no estabelecimento 1, a retenção nas peças não diferiu entre as posições. Por outro lado, nas peças adquiridas no estabelecimento 2 do mesmo município, a retenção na posição 3 (interna) diferiu das demais, apresentando as menores retenções.

Nos moirões adquiridos no estabelecimento 1, observaram-se diferenças entre as retenções de CCA para as três posições analisadas, tendo a posição 2 apresentado a maior retenção, enquanto na posição 3 foi observada a menor retenção. Contudo, os moirões comercializados nesse estabelecimento atenderam aos parâmetros da NBR 9480 (ABNT, 2009) para todas as posições analisadas.

As retenções do preservante nas peças oriundas dos estabelecimentos 4 e 5 não diferiram estatisticamente entre as amostras provenientes das posições externa e mediana dos discos, tendo essas amostras apresentado maiores retenções do CCA do que as amostras provenientes da porção interna do disco. Já para as peças provenientes do estabelecimento 6, localizado no mesmo município, não se observou diferença significativa entre as retenções das amostras de madeira provenientes das 
Tabela 5. Comparações entre médias de retenção do CCA $\left(\mathrm{kg}\right.$ i.a.m $\left.{ }^{-3}\right)$ para as posições nos moirões para cada estabelecimento.

Table 5. Comparisons among averages CCA retention $\left(\mathrm{kg}\right.$ i.a. $\left.\mathrm{m}^{-3}\right)$ to positions on fence posts for each establishment.

\begin{tabular}{cccc} 
Estabelecimento & \multicolumn{3}{c}{ Retenção $\left(\mathbf{k g}\right.$ i.a.m $\left.\mathbf{3}^{-\mathbf{3}}\right)$} \\
\cline { 2 - 4 } & $\mathbf{1}$ (Externa) & $\mathbf{2}$ (Mediana) & $\mathbf{3}$ (Interna) \\
\hline 1 & $8,36 \pm 1,55^{\mathrm{Abc}}$ & $7,31 \pm 1,96^{\mathrm{Ab}}$ & $8,69 \pm 2,22^{\mathrm{Aa}}$ \\
\hline 3 & $8,66 \pm 1,51^{\mathrm{Abc}}$ & $6,72 \pm 2,83^{\mathrm{Abc}}$ & $0,65 \pm 0,74^{\mathrm{Bb}}$ \\
4 & $12,46 \pm 2,25^{\mathrm{ABab}}$ & $14,37 \pm 6,66^{\mathrm{Aa}}$ & $7,72 \pm 6,52^{\mathrm{Ba}}$ \\
\hline 5 & $15,33 \pm 2,41^{\mathrm{Aa}}$ & $11,06 \pm 7,03^{\mathrm{Aab}}$ & $1,63 \pm 2,78^{\mathrm{Bb}}$ \\
\hline 6 & $13,19 \pm 3,13^{\mathrm{Aab}}$ & $7,97 \pm 1,55^{\mathrm{Ab}}$ & $2,07 \pm 3,22^{\mathrm{Bb}}$ \\
\hline
\end{tabular}

Médias seguidas da mesma letra, maiúscula na horizontal ou minúscula na vertical, não diferem estatisticamente pelo testes de Duncan $(p \geq 0,05)$.

três posições analisadas no disco; contudo, os valores encontrados estão abaixo do recomendado pela NBR 9480 (ABNT, 2009).

Para as amostras provenientes da posição 1 (externa) do disco, a retenção nas peças adquiridas no estabelecimento 4 diferiu estatisticamente daquelas obtidas nos moirões provenientes dos estabelecimentos 1, 2 e 6, apresentando, juntamente com os moirões adquiridos nos estabelecimentos 3 e 5 , as maiores médias de retenção do CCA. Por outro lado, as peças oriundas do estabelecimento 6 apresentaram a menor média de retenção, sendo inferior à retenção mínima indicada pela NBR 9480 (ABNT, 2009), para peças instaladas em contato direto com o solo.

$\mathrm{Na}$ posição 2 (mediana), a retenção nas peças provenientes do estabelecimento 3 diferiu estatisticamente das demais, tendo, juntamente com as peças do estabelecimento 4 , as maiores retenções e a madeira adquirida no estabelecimento 6 , a menor retenção.

Na posição 3 (interna), observou-se que, para os valores médios de retenção, os moirões adquiridos nos estabelecimentos 4, 5 e 6 (Cachoeiro de Itapemirim) e 2 (Alegre) não diferiram estatisticamente, tendo apresentado as menores retenções, enquanto as peças provenientes dos estabelecimentos 1 (Alegre) e 3 (Jerônimo Monteiro) apresentaram as maiores retenções, sendo superiores ao recomendado pela NBR 9480 (ABNT, 2009). Isto é vantajoso, pois, no caso de rachaduras em peças instaladas no campo, não haveria a exposição de madeira tratada de forma inadequada, o que poderia favorecer o ataque e o desenvolvimento de organismos xilófagos.
Em função dos resultados obtidos, seria interessante a realização de testes para avaliar o desempenho dos moirões preservados comercializados nos municípios de Alegre, Jerônimo Monteiro e Cachoeiro de Itapemirim, por meio de testes com fungos e cupins, em níveis de laboratório e de campo.

\section{CONCLUSÕES}

O produto empregado no tratamento dos moirões comercializados em três cidades (seis estabelecimentos) do sul do Estado do Espírito Santo foi o CCA.

Os moirões comercializados em seis estabelecimentos do Estado do Espírito Santo atendem aos parâmetros da Norma Técnica quanto à uniformidade da distribuição do CCA nas peças. Porém, para a penetração do CCA, alguns dos moirões comercializados nos estabelecimentos $4,6 \mathrm{e}$ 2 (Cachoeiro de Itapemirim e Alegre) não atendem aos requisitos mínimos da Associação Brasileira de Normas Técnicas.

Os moirões comercializados nos estabelecimentos $1,3,4$ e 5 atendem à retenção mínima estabelecida pela normatização para peças tratadas com produtos hidrossolúveis a serem utilizadas em contato direto com o solo. Contudo, os moirões dos estabelecimentos 2 e 6 não atendem às especificações da normatização brasileira.

Os moirões de eucalipto comercializados no município de Jerônimo Monteiro apresentam a melhor qualidade do tratamento e aqueles comercializados no estabelecimento 6 (Cachoeiro 
de Itapemirim) apresentam proteção insatisfatória para os testes executados, conforme os parâmetros da Associação Brasileira de Normas Técnicas.

\section{STATUS DA SUBMISSÃO}

Recebido: 22/03/2012

Aceito: $10 / 07 / 2012$

Publicado: 31/12/2012

\section{AUTOR(ES) PARA CORRESPONDÊNCIA}

\section{Juarez Benigno Paes}

Laboratório de Biodeterioração da Madeira - LBM, Departamento de Ciências Florestais e da Madeira - DCFM, Universidade Federal do Espírito Santo - UFES, Av. Governador Lindemberg, 316, Centro, CEP 29550-000, Jerônimo Monteiro, ES, Brasil e-mail: jbp2@uol.com.br

\section{REFERENNCIAS}

Associação Brasileira de Normas Técnicas - ABNT. NBR 6232: penetração e retenção de preservativos em postes de madeira. Rio de Janeiro: ABNT; 1973. 12 p.

Associação Brasileira de Normas Técnicas - ABNT. NBR 9480: peças roliças preservadas de eucalipto para construções rurais - Requisitos. Rio de Janeiro: ABNT; 2009. 12 p.

Farias Sobrinho DW, Paes JB, Furtado DA. Tratamento preservativo da madeira de algaroba (Prosopis juliflora
(Sw) D.C.), pelo método de substituição de seiva. Cerne 2005; 11(3): 225-236.

Freitas VP. Variações na retenção de CCA-A em estacas de Pinus após 21 anos de exposição em campo de apodrecimento [dissertação]. Piracicaba: Escola Superior de Agricultura "Luiz de Queiroz", Universidade de São Paulo; 2002. 64 p.

Galvão APM. Característica da distribuição de alguns preservativos hidrossolúveis em moirões roliços de Eucalyptus alba Reinw., tratados pelo processo de absorção por transpiração radial [tese]. Piracicaba: Escola Superior de Agricultura "Luiz de Queiroz", Universidade de São Paulo; 1968. 115 p.

Geraldo FC. Mourões para cerca: a renovação necessária. Remade; 2010. 2 p. [cited 2012 abr. 17]. Available from: http://www.remade.com.br/br/artigos_tecnicos.php.

Moreschi JC. Ensaios biológicos: uma nova alternativa para a determinação dos ingredientes ativos do preservativo CCA e estudos de interações [tese]. Curitiba: Universidade Federal do Paraná; 1985. 128 p.

Paes JB. Viabilidade do tratamento preservativo de moirões de bracatinga (Mimosa scabrella Benth.), por meio de métodos simples e comparações de sua tratabilidade com a do Eucalyptus viminalis Lab [dissertação]. Curitiba: Universidade Federal do Paraná; 1991. 140 p.

Paes JB, Moreschi JC, Lelles JG. Avaliação do tratamento preservativo de moirões de Eucalyptus viminalis Lab. e de bracatinga (Mimosa scabrella Benth.) pelo método de substituição da seiva. Ciência Florestal 2005; 15(1): 75-86.

Torres PMA, Paes JB, Lira Filho JA, Nascimento JWB. Tratamento preservativo da madeira juvenil de Eucalyptus camaldulensis Dehnh. pelo método de substituição de seiva. Cerne 2011; 17(2): 275-282. 